\title{
Mindfulness-Based Intervention on Distressing Hallucination-Like Experiences in a Nonclinical Sample
}

\author{
Álvaro I. Langer,' Adolfo J. Cangas,' and José Gallego² \\ ' Department of Clinical Psychology, University of Almeria, Spain \\ 2 Department of Social Sciences, Language, Literature, Physical Education and Sport, University of \\ Almeria, Spain
}

\begin{abstract}
The presence of psychotic-like experiences in the general population has been amply reported. Nevertheless, the degree of concern or anxiety that such experiences may generate is an aspect that has not received as much appraisal. In this sense, mindfulness is an approach to intervention based on the modification of the individual's relationship with the symptoms, instead of their elimination. The goal of the present study is to compare the effect of mindfulness training on distressing hallucination-like experiences. Eighteen participants were assigned to the experimental group, and they received 8 sessions of mindfulness training; 20 participants were assigned to a control group that viewed 8 sessions of a video forum. The results showed that upon completing the mindfulness training, there was a significant and large effect on the decrease of anxiety caused by hallucinationlike experiences. These results were maintained at the 16-week follow-up. The repercussions of these results are underlined.
\end{abstract}

Keywords: mindfulness, hallucination-like experiences, meditation, predisposition to hallucinations

The presence of psychotic-like experiences in the general population has been amply reported in diverse studies (Barret \& Etheridge, 1992; Bentall \& Slade, 1985; Johns \& van Os, 2001; Morrison, 1998; Ohayon, 2000; Serper, Dill, Chang, Kot, \& Elliot, 2005; Slade \& Bentall, 1988; Tien, 1991; Verdoux \& van Os, 2002). However, most of the works have focused mainly on the assessment of the presence or absence of diverse symptoms, without assessing other aspects that may be relevant to the experiences (such as the attributions made, the degree of discomfort or distress caused, interference in daily life, and so on). But, just as it is important to differentiate the types of behaviours carried out and their diverse prognostic power for the development of psychopathological disorders (Cangas, Langer, \& Moriana, in press; Yung et al., 2009), it is also essential to attend to the diverse psychological mechanisms involved in the response to the experience, which would be influenced by beliefs and

Address for correspondence: Álvaro I Langer. Universidad de Almería. La Cañada de San Urbano s/n. 04120 Almería (Spain).E-mail: alh750@ual.es 
appraisals (Bentall, Haddock, \& Slade, 1994; Chadwick, Birchwood, \& Trower, 1996; Morrison, 2001), especially the degree of discomfort and worry, which differentiates healthy and unhealthy individuals (Peters, Days, McKenna, \& Orbach, 1999).

In this connection, interventions based on mindfulness (Kabat-Zinn, 1990; Segal, Williams, \& Teasdale, 2002) are a promising treatment in the approach to diverse pathologies (Baer, 2003). One of the pillars of mindfulness is that the feeling of distress is not inherent to a private event, but instead is caused by the way one relates to the experience (Segal et al., 2002), which is mainly to avoid it or to attempt to suppress it. However, these coping styles will paradigmatically increase the frequency and distress of the symptoms (García-Montes, Pérez-Álvarez, \& Fidalgo, 2003; Salkovsky \& Campbell, 1994).

Eliminating the distress associated with psychotic symptoms has been the focus of the studies that have applied mindfulness in psychosis (Abba, Chadwick, \& Stevenson, 2008; Chadwick, Newman-Tylor, \& Abba, 2005; Chadwick, Hughes, Russell, Russell, \& Dagnan, 2009; Davis, Strasburger, \& Brown, 2007). The results of these initial studies have mainly reached two conclusions: mindfulness intervention, which has not shown iatrogenic effects, can be applied in psychosis, and it would have a positive effect on the reduction of the associated symptoms such as stress and anxiety, increasing the sense of control over the experiences and the sense of personal empowerment. Nevertheless, these first works (Chadwick et al., 2009) have either not used a control group that included some kind of attention to the patients' wait-list or else they were based on qualitative analysis of the data (Abba et al., 2008; Davis et al., 2007). Nor did they use any follow-up to determine whether the results were long lasting.

Along these lines, the goal of the present study was to determine whether mindfulness training has an effect on psychotic-like experiences; in particular, the components associated with anxiety or distress in these types of experiences. The hypothesis is that mindfulness may decrease the discomfort and anxiety due to the experiences but it will not necessarily decrease the frequency of the experiences.

\section{Method}

\section{Participants and Design}

The participants were students from the University of Almería, Spain, who scored positively on the Hallucinations Revised Scale and who also reported that these experiences caused them distress or anxiety higher than or equal to 5 (on a scale ranging from 1 to 10 ). Participants who did not meet these criteria were excluded.

From an initial sample of 123 students, 63 met the established criteria. Out of these, 38 completed the entire study. Twenty in the control group (18 women, 2 men), ages between 19 and 26 years $(M=21.05, S D=1.82)$ and 18 in the experimental group (14 women, 4 men), ages between 18 and 32 years $(M=21.56, S D=3.33)$.

The participants were assigned to the groups in a quasi-random procedure: the first student was assigned to the experimental group, the second one to the control group, and so on. The participants received extra credits in a subject for participating in the study.

\section{Materials}

Revised Hallucination Scale (RHS; Morrison, Wells, $\mathcal{E}$ Nothard, 2000). This scale was designed based on the Launay-Slade Hallucination Scale (Launay \& 
Slade, 1981), which measures predisposition to hallucinations in normal population. It consists of thirteen 4-point Likert-type formatted items, ranging from 1 (Never) to 4 (Almost always). The psychometric properties of the Spanish version were published by Cangas et al. (in press), who found a four-factor structure by means of principal component analysis (PCA). These four factors accounted for $52.8 \%$ of the variance $(24.4,11.5,9.7$ and $8.3 \%$, respectively). Internal consistency of the scale as measured by Cronbach's alpha coefficient was .70. For this study, participants were requested to indicate, on the one hand, the degree of distress produced by each item described in the RHS, and, on the other hand, the degree of anxiety. Both were quantified on a scale ranging from 1 to 10 . When participants responded Never to an item, they went directly on to the next item.

\section{Procedure}

The groups were made up of 8 to 10 participants. In each group, the procedure was as follows.

\section{Experimental Group}

The training consisted of eight 1-hour sessions of mindfulness-based cognitive therapy (MBCT) (Segal et al., 2002). First, they were trained in Body Scan, mindful breathing, breathing space, yoga (stretching exercises), and sitting meditation. At the end of the session, they received handouts with a summary of the session. In addition, they received a CD with body scan and sitting meditation and homework completion forms.

\section{Control Group}

The control group (placebo) consisted of eight 1-hour sessions of viewing a videoforum. The sessions included various films and short films of diverse social and cultural topics such as education, immigration, marginality, family, etc. Towards the end of the session, the participants were invited to express their opinion.

\section{Follow-Up}

16 weeks after completing the treatment, the participants were summoned to fill in once again the same questionnaires that had been administered at the first session and after completing the training.

\section{Results}

To analyse the results obtained, we calculated the differences of means with Student's $t$ for independent samples and compared posttreatment with pretreatment, and subsequently, follow-up with pretreatment. Likewise, effect size of change (Cohen's d) was calculated.

\section{Immediate Effects}

When comparing the experimental group and control group scores on anxiety, a significant difference with a large effect size was observed between the groups. In the remaining variables, no significant differences were obtained, although there were some important changes in distress and in intrusive thoughts and auditory distortions (which did not reach statistical significance; see Table 1). 


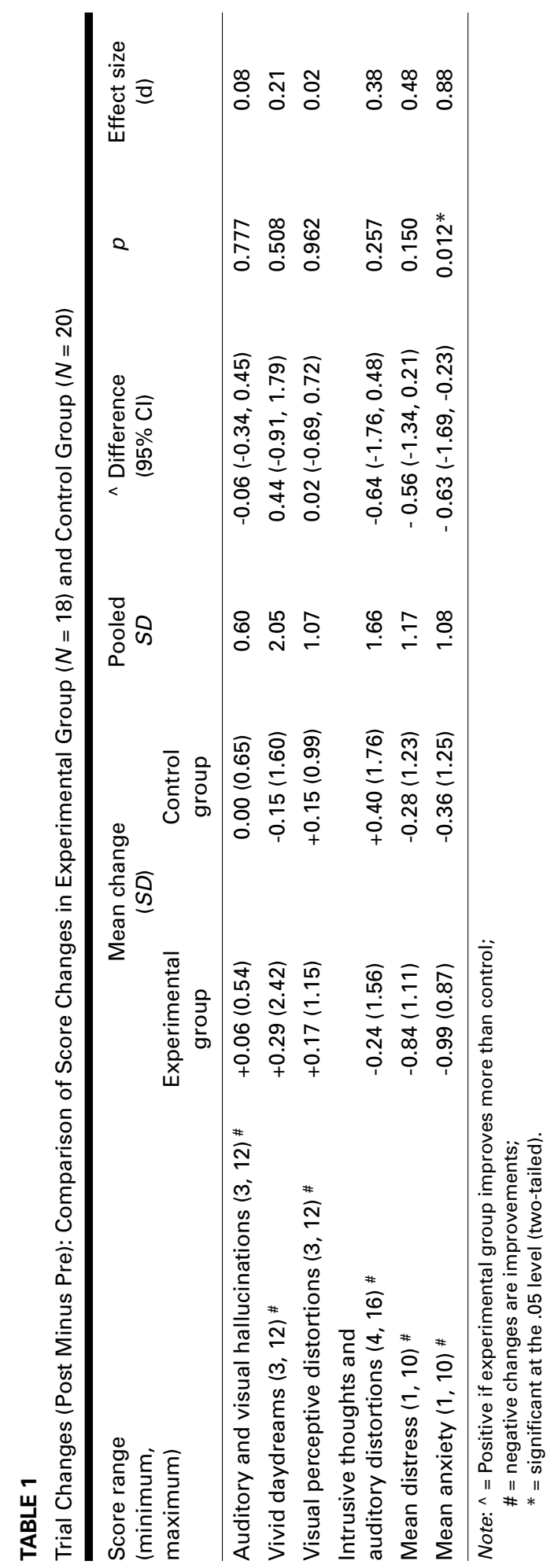




\section{Long-Term Effects}

At follow-up, all the participants from both groups who completed the intervention were summoned, and $63.16 \%$ of the initial sample complied: $78 \%$ of the experimental group and $50 \%$ of the control group. When comparing the baseline results with those at follow-up, the statistically significant differences in anxiety observed upon completing treatment were maintained, and there was a notable difference in the variable distress (although it was statistically nonsignificant). In the variables Intrusive thoughts and Auditory distortions, the differences were barely noticeable (see Table 2).

\section{Discussion}

In the present study, we analysed the effect of MBCT on hallucination-like experiences. To date, the only work that had presented a controlled study with clinical sample was that of Chadwick et al. (2009), who found that all the scores were in the expected direction, but they were not higher than those of the control group. In this work, we found that anxiety was significantly lower in the experimental group compared to the control group. The effect size of the results is comparable to a recent study that assessed the effect of mindfulness in a group of students with obsessive symptoms (Hanstede, Gidron, \& Nyklicek, 2008). It should be taken into account that anxiety has been postulated to occur at the onset and maintenance of hallucinations (Freeman \& Garety, 2003; Morrison, 1998, 2001); in fact, anxiety was the focus of a mindfulness intervention in a group of five psychotic patients (Davis et al., 2007).

With regard to the initial hypothesis, on the one hand, as expected, there was no significant decrease in the frequency, and there was even a slight increase in some factors. This behaviour is coherent with the therapeutic models that use mindfulness and acceptance (Hayes, Follette, \& Linehan, 2004), in which decrease in frequency is not the goal of intervention, but instead a change in the function, that is, in the relationship that the individual establishes with the symptom or private event. Thus it was shown in the pioneer study carried out by Bach and Hayes (2002) with patients with psychosis, in which the experimental group had fewer relapses despite an increase in the symptomatology. Our hypothesis was partially confirmed. Distress and anxiety were decreased with the intervention, but only anxiety was significantly lower in the experimental group at post-treatment and followup in comparison to the control group. However, it is important to determine whether significant differences would be observed if other control groups (i.e., waiting-list) were used. In that case, it could be concluded that this variable depends, to a great extent, on the attention the participants received (although it is unspecific), and thus, their results are closely related to the placebo effect.

With regard to the maintenance of the results, this is the first study to perform a follow-up in an intervention with hallucination and hallucination-like experiences. The results were maintained 16 weeks after completing the intervention. Therefore, the effects of the treatment are long lasting, at least in the follow-up that was carried out.

In other words, it is suggested that MBCT training, that is, letting any thought, emotion, or physical feeling arise in awareness and not react automatically - letting thoughts go, considering them as just thoughts and not events - allows people to distance themselves from their internal events, which leads to a change in the function of the experience, in this case, reducing the anxiety it provokes. 


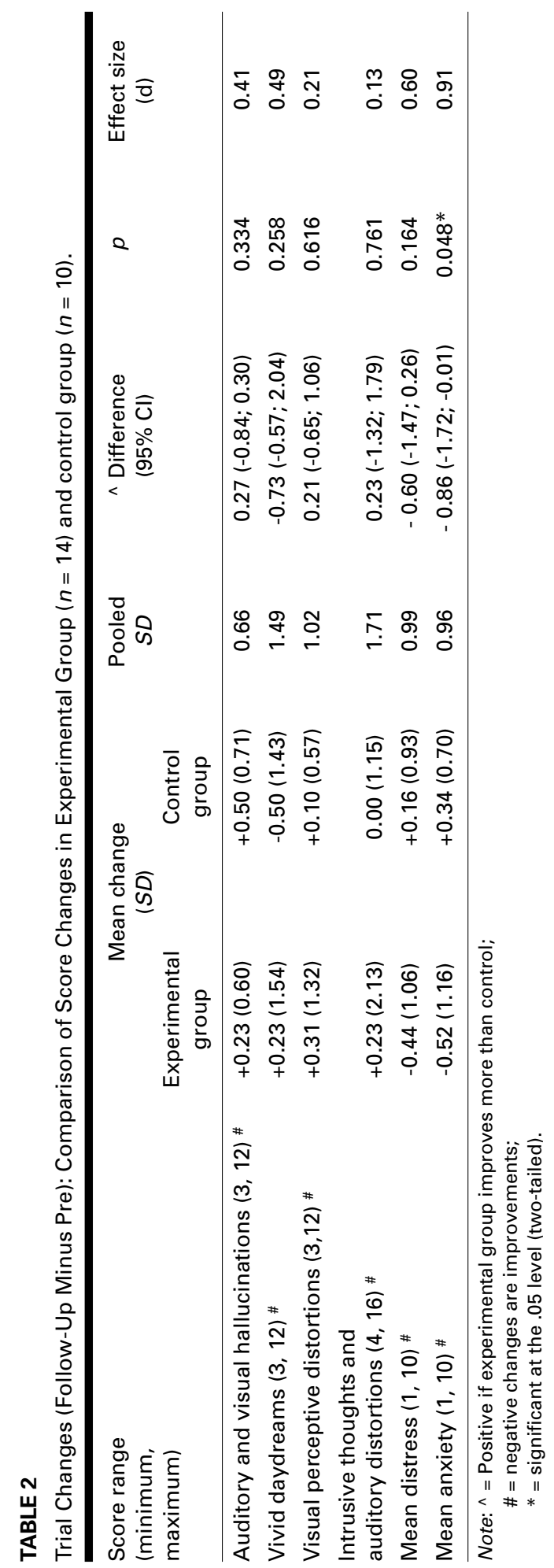


In accordance, we consider these results promising, and they confirm that controlled interventions with mindfulness produce important results, as has been observed in psychotic patients (Abba et al., 2008; Chadwick et al., 2005; Chadwick et al., 2009). Among the limitations of the study, we note the small sample size and the need to replicate the study with clinical samples to verify whether or not the results obtained are confirmed in patients who experience hallucinations.

\section{Acknowledgments}

This work was supported by FPU Scholarship from Spanish Ministry of Science and Education (AP/2007-02810) awarded to the first author and a research project supported by the Spanish Ministry of Science and Education (SEJ2006-02342/PSIC) awarded to the second author.

\section{References}

Abba, N., Chadwick, P., \& Stevenson, C. (2008). Responding mindfully to distressing psychosis: A grounded theory analysis. Psychotherapy Research, 18, 77-87

Bach, P., \& Hayes, S.C. (2002). The use of Acceptance and Commitment Therapy to prevent the rehospitalization of psychotic patients: A randomized controlled trail. Journal of Consulting and Clinical Psychology, 70, 1129-1139.

Baer, R.A. (2003). Mindfulness training as a clinical intervention: A conceptual and empirical review. Clinical Psychology: Science and Practice, 10, 125-143.

Barret, T.R., \& Etheridge, J.B. (1992). Verbal hallucinations in normals I: People who hear 'voices'. Applied Cognitive Psychology, 6, 379-387.

Bentall, R.P., \& Slade P.D. (1985). Realibility of a scale measuring disposition towards hallucinations: a brief report. Personality and Individual Differences, 6, 527-529.

Bentall, R.P., Haddock, G., \& Slade, P.D. (1994). Pychological treatment for auditory hallucinations: From theory to therapy. Behavior Therapy, 25, 51-66.

Cangas, A.J., Langer, A.I., \& Moriana, J.A. (in press). Hallucinations and related perceptual disturbance in a non-clinical spanish population. International Journal of Social Psychiatry.

Chadwick, P., Birchwood, M., \& Trower, P. (1996). Cognitive therapy for delusions, voices and paranoia. New York: Wiley.

Chadwick, P., Newman-Tylor, K., \& Abba, N. (2005). Mindfulness groups for people whit distressing voices. Behavioural and Cognitive Psychotherapy, 33, 351-359.

Chadwick, P., Hughes, S., Russell, D., Russell, I., \& Dagnan, D. (2009). Mindfulness for distressing voices and paranoia: A replication and randomized feasibility trial. Behavioural and Cognitive Psychotherapy, 37, 403-412.

Davis, L.W., Strasburger, A.M., \& Brown, L.F. (2007). Mindfulness: an intervention for anxiety in schizopherenia. Journal of Psychosocial Nursing and Mental Health Service, 45, 22-29.

Freeman, D., \& Garety, P. A. (2003). Connecting neurosis and psychosis: the direct influence of emotion on delusions and hallucinations. Behaviour Research and Therapy, 41, 923-947.

García-Montes, J.M., Pérez-Álvarez, M., \& Fidalgo, A. (2003). Influence of the suppression of selfdiscrepant thoughts on the vividness of perception of auditory illusions. Behavioural and Cognitive Psychotherapy, 31, 33-44.

Hanstede, M., Gidron, Y., \& Nyklicek, I. (2008). The effects of a mindfulness intervention on obsessive-compulsive symptoms in a non-clinical student population Journal of Nervous and Mental Disease, 196, 776-779.

Hayes, S.C., Follette, V.M., \& Linehan, M.M. (Eds.). (2004). Mindfulness and acceptance: Expanding the cognitive behavioral tradition. New York: The Guilford Press.

Johns, L.C., \& van Os, J. (2001). The continuity of psychotic experiences in the general population. Clinical Psychology Review, 21, 1125-1141. 
Kabat-Zinn, J. (1990). Full catastrophe living: Using the wisdom of your body and mind to face stress, pain and illness. New York: Hyperion.

Launay, G., \& Slade, P.D. (1981). The measurement of hallucinatory predisposition in male and female prisoners. Personality and Individual Differences, 2, 221-234.

Morrison, A.P. (1998). A cognitive analysis of auditory hallucinations: Are voices to schizophrenia what bodily sensations are to panic? Behavioural and Cognitive Psychotherapy, 26, 289-302.

Morrison, A.P. (2001). The interpretation of intrusions in psychosis: An integrative cognitive approach to hallucinations and delusions. Behavioural and Cognitive Psychotherapy, 29, 257-276.

Morrison, A.P., Wells, A., \& Nothard, S. (2000). Cognitive factors in predisposition to auditory and visual hallucinations. British Journal of Clinical Psychology, 39, 67-78.

Ohayon, M.M. (2000). Prevalence of hallucinations and their pathological associations in the general population. Psychiatry Research, 97, 153-164.

Peters, E., Days, S., McKenna, J., \& Orbach, G. (1999). Delusional ideation in religious and psychotic populations. British Journal of Clinical Psychology, 38, 83-96.

Salkoviskis, P. M., \& Campbell, P. (1994). Thought suppression induces intrusion in naturally occurring negative intrusive thoughts. Behaviour Research and Therapy, 32, 1-8

Segal, Z., Williams, J., \& Teasdale, J. (2002). Mindfulness based cognitive therapy for depression. New York: Guilford.

Serper, M., Dill, C.A., Chang, N., Kot, T., \& Elliot, J. (2005). Factorial structure and classification of hallucinations: Continuity of experience in psychotic and normal individuals. Journal of Nervous and Mental Disease, 193, 265-272.

Slade, P.D., \& Bentall, R.P. (1988). Sensory deception: A scientific analysis of hallucination. Baltimore: Johns Hopkins University.

Tien, A. (1991). Distributions of hallucinations in the population. Social Psychiatry and Psychiatric Epidemiology, 26, 287-292.

Verdoux, H., \& van Os, J. (2002). Psychotic symptoms in non-clinical populations and the continuum of psychosis. Schizophrenia Research, 54, 59-65.

Yung, A.R., Nelson, B., Baker, K., Buckby, J.A., Baksheev, G., \& Cosgrave, E.M. (2009). Psychotic-like experiences in a community sample of adolescents: Implications for the continuum model of psychosis and prediction of schizophrenia. Australian and New Zealand Journal of Psychiatry, 43, 118-128. 